\title{
Mathematical Solvability of a Caputo Fractional Polymer Degradation Model Using Further Generalized Functions
}

\author{
Emile Franc Doungmo Goufo and Stella Mugisha \\ Department of Mathematical Sciences, University of South Africa, Florida Science Campus, Florida, Gauteng 0003, South Africa \\ Correspondence should be addressed to Emile Franc Doungmo Goufo; dgoufef@unisa.ac.za
}

Received 12 May 2014; Accepted 10 June 2014; Published 25 June 2014

Academic Editor: Abdon Atangana

Copyright ( 2014 E. F. Doungmo Goufo and S. Mugisha. This is an open access article distributed under the Creative Commons Attribution License, which permits unrestricted use, distribution, and reproduction in any medium, provided the original work is properly cited.

\begin{abstract}
The continuous fission equation with derivative of fractional order $\alpha$, describing the polymer chain degradation, is solved explicitly. We prove that, whether the breakup rate depends on the size of the chain breaking up or not, the evolution of the polymer sizes distribution is governed by a combination of higher transcendental functions, namely, Mittag-Leffler function, the further generalized G-function, and the Pochhammer polynomial. In particular, this shows the existence of an eigenproperty; that is, the system describing fractional polymer chain degradation contains replicated and partially replicated fractional poles, whose effects are given by these functions.
\end{abstract}

\section{Introduction}

Polymer degradation is the process where polymers are converted into monomers or mixtures of monomers. Polymers range from familiar synthetic plastics such as polystyrene (also called styrofoam) to natural biopolymers such as DNA and proteins that are fundamental to biological structure and function. Historically, products arising from the linkage of repeating units by covalent chemical bonds have been the primary focus of polymer science; emerging important areas of the science now focus on noncovalent links. Polyisoprene of latex rubber and the polystyrene of styrofoam are examples of polymeric natural/biological and synthetic polymers, respectively. In biological contexts, essentially, all biological macromolecules, that is, proteins (polyamides), nucleic acids (polynucleotides), and polysaccharides, are purely polymeric and are composed in large part of polymeric components, for instance, isoprenylated/lipid-modified glycoproteins, where small lipidic molecule and oligosaccharide modifications occur on the polyamide backbone of the protein. In the theory of polymers division, one would expect a conservation of mass, especially when polymers are converted into monomers or mixtures of monomers, but $[1,2]$ an infinite cascade of division events creating a "dust" of monomers of zero size carrying nonzero mass and leading to nonconservativeness (dishonesty) in the model has been observed. Since this remains partially unexplained by classical models of clusters' fission, extending the analysis to the fractional version and expressing the solutions explicitly may bring more light and a broader outlook about this phenomenon which remains a mystery. Thus, we are going to analyze the fractional fission system describing the polymer chain degradation and provide explicit expressions of its solutions. Before that, let us have a look at what is known about the classical kinetics of polymer chain degradation. However, there is a growing interest in extending the normal calculus with integer orders to noninteger orders (real or complex order) [3-6] because its applications, like, for example, the topic of this paper, have caught a great range of consideration in the past decade. For instance, in [3] the authors made use of the homotopy decomposition method (HDM), to solve a system of fractional nonlinear differential equations that arise in the model for HIV infection of CD4+ T cells and attractor one-dimensional Keller-Segel equations. In [4], two methods including Frobenius and Adomian decomposition method were used to generalize the classical Darcy law by regarding the water flow as a function of a noninteger order derivative of the piezometric head. 
1.1. The Classic Polymer Degradation Dynamics. The binary fission integrodifferential equation,

$$
\begin{aligned}
\frac{\partial}{\partial t} g(x, t)= & -g(x, t) \int_{0}^{x} H(y, x-y) d y \\
& +2 \int_{x}^{\infty} g(y, t) H(x, y-x) d y, \quad x, t>0,
\end{aligned}
$$

is the kinetic equation describing the evolution of the sizes distribution. Here, $g(x, t)$ represents the density of $x$-groups (i.e., groups of size $x)$ at time $t$, and $H(x, y)$ gives the average fission rate, that is, the average number at which clusters of size $x+y$ undergo splitting to form an $x$-group and a $y$-group. This model is applicable in many branches of natural sciences ranging from physics, through chemistry, engineering, and biology, to ecology and in numerous domains of applied sciences, the rock fractures, and break of droplets. Various types of fragmentation equations have been comprehensively analyzed in numerous works (see, e.g., [2, 7-11]). In the domain of polymer science, the fission dynamics have also been of considerable interest, since degradation of bonds or depolymerisation results in fragmentation; see [12-14]. In [13], the authors used the statistical arguments to find and analyse the size distribution of the model. The authors in [12] analysed the model in combination with the inverse process, that is, the coagulation process, and provided a similar result for the size distribution.

\section{Fractional Fission Differential Problem}

We aim to investigate the evolution of the number density of particles described by the fractional fission integrodifferential equation

$$
\begin{aligned}
D_{t}^{\alpha} g(x, t)= & -g(x, t) \int_{0}^{x} H_{\alpha}(y, x-y) d y \\
& +2 \int_{x}^{\infty} g(y, t) H_{\alpha}(x, y-x) d y, \\
& 0 \leq \alpha<1, x, t>0,
\end{aligned}
$$

subject to the following initial condition:

$$
g(x, 0)=f(x), \quad x, t>0,
$$

where

$$
\begin{aligned}
{ }_{0}^{C} D_{t}^{\alpha} g(x, t) & =\frac{\partial^{\alpha}}{\partial t^{\alpha}} g(x, t) \\
& =\frac{1}{\Gamma(1-\alpha)} \int_{0}^{t}(t-r)^{-\alpha} \frac{\partial}{\partial r} g(x, r) d r,
\end{aligned}
$$

where $0 \leq \alpha<1$ is the fractional derivative of $g(x, t)$ in the sense of Caputo [15], with $\Gamma$ the Gama function. For reasons of simplicity, we note that ${ }_{0}^{C} D_{t}^{\alpha}=D_{t}^{\alpha}$.

\section{Mathematical Analysis}

Our analysis consists of two distinct cases: the case where the breakup rate depends on the size of the chain breaking up and the case where it does not depend. This will help us compare and analyse the two scenarios.

3.1. The Case $H_{\alpha}(x, y)=1$. Firstly we assume that the rate of breakup is independent of the length of polymer. Model (2) becomes

$$
D_{t}^{\alpha} g(x, t)=-x g(x, t)+2 \int_{x}^{\infty} g(y, t) d y, \quad 0 \leq \alpha<1 ;
$$

applying the Laplace transform on both sides of the latter equation yields

$$
\mathscr{L}\left(D_{t}^{\alpha} g(x, t), s\right)=\mathscr{L}\left(-x g(x, t)+2 \int_{x}^{\infty} g(y, t) d y, s\right) .
$$

Clearly,

$$
\begin{gathered}
\mathscr{L}\left(D_{t}^{\alpha} g(x, t), s\right)=s^{\alpha} \tilde{g}(x, s)-s^{\alpha-1} f(x) \\
\mathscr{L}\left(-x g(x, t)+2 \int_{x}^{\infty} g(y, t) d y\right)= \\
-x \tilde{g}(x, s) \\
+2 \int_{x}^{\infty} \tilde{g}(y, s) d y
\end{gathered}
$$

where $\tilde{g}(x, s)$ is the Laplace transform $\mathscr{L}(g(x, t), s)$. We obtain

$$
f(x)=\left(\frac{x}{s^{\alpha-1}}+s\right) \tilde{g}(x, s)-\frac{2}{s^{\alpha-1}} \int_{x}^{\infty} \tilde{g}(y, s) d y .
$$

Remark 1. We note that by the differential expression (5) we implicitly require that $y \rightarrow g(y, t)$ should be Lebesgue integrable on any $[\epsilon, \infty)$ for $\epsilon>0$ and almost every $x>0$. The same assumption therefore applies to $y \rightarrow f(y)$ and $y \rightarrow \widetilde{g}(y, s)$.

Hence we put $Y(x, s)=\left(2 / s^{\alpha-1}\right) \int_{x}^{\infty} \tilde{g}(y, s) d y$ with the confidence that the integrand is integrable over any interval $[\epsilon, \infty)$ so that the integral is absolutely continuous at each $x>$ 0 and we can thus differentiate so as to convert (8) into the partial differential equation as follows:

$$
f(x)=\left(\frac{x}{s^{\alpha-1}}+s\right) \frac{s^{\alpha-1}}{2} \partial_{x} Y(x, s)-Y(x, s) .
$$

Choosing the constant in the general solution so as to have solutions converging to zero at $\infty$, we obtain its solution which is given as

$$
Y(x, s)=2 e^{-\xi_{s, \alpha}(x)} \int_{x}^{\infty} e^{\xi_{s, \alpha}(\eta)} \frac{f(\eta)}{\eta+s^{\alpha}} d \eta,
$$

where

$$
\xi_{s, \alpha}(x)=\int_{0}^{x} \frac{2}{\eta+s^{\alpha}} d \eta=\ln \left(\frac{x+s^{\alpha}}{s^{\alpha}}\right)^{2} .
$$


This leads to the solution of (8) as follows:

$$
\begin{aligned}
\tilde{g}(x, s) & =\frac{s^{\alpha-1} f(x)}{x+s^{\alpha}}+\frac{2 s^{\alpha-1}}{x+s^{\alpha}} e^{-\xi_{s, \alpha}(x)} \int_{x}^{\infty} e^{\xi_{s, \alpha}(\eta)} \frac{f(\eta)}{\eta+s^{\alpha}} d \eta \\
& =\frac{s^{\alpha-1} f(x)}{x+s^{\alpha}}+2 \frac{s^{\alpha-1}}{\left(x+s^{\alpha}\right)^{3}} \int_{x}^{\infty}\left(\eta+s^{\alpha}\right) f(\eta) d \eta
\end{aligned}
$$

Applying the inverse Laplace transform $\mathscr{L}^{-1}(\tilde{g}(x, s), t)=$ $g(x, t)$ to the latter expression yields

$$
\begin{aligned}
\mathscr{L}^{-1}\left(\frac{s^{\alpha-1} f(x)}{x+s^{\alpha}}, t\right) & =f(x) \mathscr{L}^{-1}\left(\frac{s^{\alpha-1}}{x+s^{\alpha}}, t\right) \\
& =f(x) \sum_{n=0}^{\infty} \frac{(-x)^{n}(t)^{n \alpha}}{\Gamma(n \alpha+1)} \\
& =f(x) E_{\alpha}\left[-x t^{\alpha}\right],
\end{aligned}
$$

where $E_{\alpha}$ is the Mittag-Leffler function as follows:

$$
E_{\alpha}[x]=\sum_{n=0}^{\infty} \frac{x^{n}}{\Gamma(n \alpha+1)} .
$$

We also have

$$
\begin{array}{r}
\mathscr{L}^{-1}\left(2 \frac{s^{\alpha-1}}{\left(x+s^{\alpha}\right)^{3}} \int_{x}^{\infty}\left(\eta+s^{\alpha}\right) f(\eta) d \eta, t\right) \\
=2 \int_{x}^{\infty} f(\eta) \mathscr{L}^{-1}\left(\frac{s^{\alpha-1}\left(\eta+s^{\alpha}\right)}{\left(x+s^{\alpha}\right)^{3}}, t\right),
\end{array}
$$

and, clearly,

$$
\begin{aligned}
\mathscr{L}^{-1} & \left(\frac{s^{\alpha-1}\left(\eta+s^{\alpha}\right)}{\left(x+s^{\alpha}\right)^{3}}, t\right) \\
= & \mathscr{L}^{-1}\left(\frac{\eta s^{\alpha-1}}{\left(x+s^{\alpha}\right)^{3}}, t\right)+\mathscr{L}^{-1}\left(\frac{s^{2 \alpha-1}}{\left(x+s^{\alpha}\right)^{3}}, t\right) \\
= & \eta \sum_{j=0}^{\infty} \frac{(-3)(-4) \cdots(-j-2) x^{j} t^{(2+j) \alpha}}{\Gamma(1+j) \Gamma(\{2+j\} \alpha+1)} \\
& +\sum_{j=0}^{\infty} \frac{(-3)(-4) \cdots(-j-2) x^{j} t^{(1+j) \alpha}}{\Gamma(1+j) \Gamma(\{1+j\} \alpha+1)} .
\end{aligned}
$$

Thus the solution of fractional model (5) is given by

$$
\begin{aligned}
g(x, t)= & f(x) E_{\alpha}\left[-x t^{\alpha}\right]+2 G_{\alpha, \alpha-1,3}(-x, t) \int_{x}^{\infty} \eta f(\eta) d \eta \\
& +2 G_{\alpha, 2 \alpha-1,3}(-x, t) \int_{x}^{\infty} f(\eta) d \eta
\end{aligned}
$$

where $G$ is the higher transcendental generalized $G$-function defined by

$$
G_{q, \beta, r}(x, t)=\sum_{j=0}^{\infty} \frac{(-r)(-1-r) \cdots(1-j-r)(-x)^{j} t^{(r+j) q-\beta-1}}{\Gamma(1+j) \Gamma(\{r+j\} q-\beta)}
$$

and expressed in terms of the Pochhammer polynomial

$$
(x-1)_{n}=(x-1)(x-2) \cdots(x-n),
$$

as

$$
G_{q, \beta, r}(x, t)=\sum_{j=0}^{\infty} \frac{(r)_{j}(-x)^{j} t^{(r+j) q-\beta-1}}{\Gamma(1+j) \Gamma(\{r+j\} q-\beta)} .
$$

(See the appendix for some properties of the generalized $G$-function.) We see that putting $\alpha=1$ in solution (17) reduces to the classic first order derivative and corresponds (as well known; see [14]) to an exponential distribution in $x$. It also shows that the polymer chains fragment exponentially fast in time. However, in fractional integrodifferential theory, the generalized $G$-function is of capital importance since it carries increased time domain complexity.

3.2. The Case $H_{\alpha}(x, y)=x+y$. This case represents a process where the rate of fission increases with size. Such a process can occur when the polymers are under tenseness or in a destructive force field such as ultrasound. Model (2) becomes

$$
D_{t}^{\alpha} g(x, t)=-x^{2} g(x, t)+2 \int_{x}^{\infty} y g(y, t) d y, \quad 0 \leq \alpha<1 .
$$

As done previously, we apply the Laplace transform to have

$$
f(x)=\left(\frac{x^{2}}{s^{\alpha-1}}+s\right) \tilde{g}(x, s)-\frac{2}{s^{\alpha-1}} \int_{x}^{\infty} y \tilde{g}(y, s) d y .
$$

By Remark 1 , we can also put $Y(x, s)=$ $\left(2 / s^{\alpha-1}\right) \int_{x}^{\infty} y \tilde{g}(y, s) d y$ and then

$$
f(x)=\left(\frac{x^{2}}{s^{\alpha-1}}+s\right) \frac{s^{\alpha-1}}{2 x} \partial_{x} Y(x, s)-Y(x, s),
$$

leading to

$$
Y(x, s)=2 e^{-\xi_{s, \alpha}(x)} \int_{x}^{\infty} e^{\xi_{s, \alpha}(\eta)} \frac{\eta f(\eta)}{\eta^{2}+s^{\alpha}} d \eta,
$$

with $\xi_{s, \alpha}(x)=\int_{0}^{x}\left(2 \eta /\left(\eta^{2}+s^{\alpha}\right)\right) d \eta=\ln \left(\left(x^{2}+s^{\alpha}\right) / s^{\alpha}\right)$. The solution of (22) reads as

$$
\tilde{g}(x, s)=\frac{s^{\alpha-1} f(x)}{x^{2}+s^{\alpha}}+\frac{2 s^{\alpha-1}}{\left(x^{2}+s^{\alpha}\right)^{2}} \int_{x}^{\infty}(\eta) f(\eta) d \eta .
$$

Applying the inverse Laplace transform and following the same steps as in the previous section finally yield the solution of fractional model (21) which is given by

$$
\begin{aligned}
g(x, t)= & f(x) E_{\alpha}\left[-x^{2} t^{\alpha}\right] \\
& +2 G_{\alpha, \alpha-1,2}\left(-x^{2}, t\right) \int_{x}^{\infty} \eta f(\eta) d \eta,
\end{aligned}
$$

where $E$ and $G$ are, respectively, defined in (14) and (18). 
If we take $f(x)=\delta(x-l)$, then the latter solution becomes

$$
g(x, t)= \begin{cases}0 & \text { for } x>l \\ \delta(x-l) E_{\alpha}\left[-l^{2} t^{\alpha}\right] & \text { for } x=l \\ 2 l G_{\alpha, \alpha-1,2}\left(-x^{2}, t\right) & \text { for } x<l\end{cases}
$$

giving the compact form

$$
g(x, t)=\delta(x-l) E_{\alpha}\left[-x^{2} t^{\alpha}\right]+2 l \vartheta(l-x) G_{\alpha, \alpha-1,2}\left(-x^{2}, t\right),
$$

where 9 is the step function.

If we compare this distribution to the previous case where the breakup rate is independent of the length of polymer, we see that the second model shows a much slower production of daughter particles due to fission. This is an expected outcome given the relative behaviour of the two breakup speeds.

\section{Concluding Remarks}

We have used the model of fractional $\alpha$ th order describing the polymer chain degradation to express the solutions explicitly. We first considered the case where the rate of breakup is independent of the length of the polymer before investigating the case where the rate of fission is a function of the size of the polymer. In both cases, we found that the solutions are given by a combination of higher transcendental functions, the Mittag-Leffler function, the further generalized $G$-function, and the Pochhammer polynomial, showing the existence in the system of repeated and partially replicated fractional poles, whose effects are given by these functions. Moreover, it is of significant usefulness to obtain here a generalized function which when fractionally differentiated or integrated (differintegrated) by any order returns itself. Like exponential, trigonometric, and hyperbolic functions of integer order calculus, the definitions of such generalized functions are important in fractional calculus, especially to describe real phenomena like the polymer chain degradation. Therefore this work extends the preceding ones, with the inclusion of fractional differentiation which was not considered before, and the results we got here, especially the further generalized $G$-function which is of capital importance since it carries increased time domain complexity.

\section{Appendix}

\section{Relevant Properties of the G-Function: (Partially) Replicated Fractional Poles}

It is obvious to see that taking $r=1$ reduces the $G$-function into the following generalized function, $R$-function [16]:

$$
R_{q, \beta}(x, t)=\sum_{j=0}^{\infty} \frac{(x)^{j} t^{(1+j) q-\beta-1}}{\Gamma(\{1+j\} q-\beta)},
$$

subject to some proportional coefficients $1 / \Gamma(1+j)=1 /(1+$ $j)$ !. Using the fractional derivative $D_{t}^{\alpha}(0<\alpha<1)$, defined in
(4), the $R$-function is proven to return itself under $\alpha$ th order differentiation. In fact we know (see [17, page 67]) that

$$
D_{t}^{\alpha}(x)^{p}=\frac{\Gamma(p+1)(x)^{p-\alpha}}{\Gamma(p-\alpha+1)}, \quad p>-1,
$$

yields

$$
D_{t}^{\alpha} R_{\alpha, 0}(x, t)=\sum_{j=0}^{\infty} \frac{(x)^{j} t^{j \alpha-1}}{\Gamma(j \alpha)} .
$$

Taking $j=k+1$ leads to

$$
D_{t}^{\alpha} R_{\alpha, 0}(x, t)=\sum_{k=-1}^{\infty} \frac{(x)^{k+1} t^{k+1 \alpha-1}}{\Gamma(k+1 \alpha)} .
$$

Thus,

$$
D_{t}^{\alpha} R_{\alpha, 0}(x, t)=x R_{\alpha, \beta}(x, t), \quad 1>\alpha>0
$$

for $t>0$. Hence for $x=1$, the function is proven to replicate, showing clearly its eigenproperty. In general, $\alpha$ th order differintegration of the $R$-function $R_{q, \beta}(x, t)$ returns another $R$-function, namely, $R_{q, \beta+\alpha}(x, t)[18]$. Special cases of the $G$-function also include the exponential function, the sine, cosine, hyperbolic sine, and hyperbolic cosine functions, and the Mittag-Leffler function, Agarwal's function, Erdelyi's function, Hartley's $F$-function, and Miller and Ross's function.

\section{Conflict of Interests}

The authors declare that there is no conflict of interests regarding the publication of this paper.

\section{References}

[1] G. T. Tsao, "Structures of cellulosic materials and their hydrolysis by enzymes," in Perspectives in Biotechnology and Applied Microbiology, D. I. Alani and M. Moo-Young, Eds., pp. 205-212, 1986.

[2] R. M. Ziff and E. D. McGrady, "Shattering transition in fragmentation," Physical Review Letters, vol. 58, no. 9, pp. 892-895, 1987.

[3] A. Atangana and E. Alabaraoye, "Solving system of fractional partial differential equations arisen in the model of HIV infection of $\mathrm{CD}^{+}$cells and attractor one-dimensional KellerSegel equation," Advances in Difference Equations, vol. 2013, article 94, 2013.

[4] A. Atangana and P. D. Vermeulen, "Analytical solutions of a space-time fractional derivative of groundwater flow equation," Abstract and Applied Analysis, vol. 2014, Article ID 381753, 11 pages, 2014.

[5] M. Caputo, "Linear models of dissipation whose $Q$ is almost frequency independent-II," Geophysical Journal of the Royal Astronomical Society, vol. 13, no. 5, pp. 529-539, 1967.

[6] I. Podlubny, Fractional Differential Equations, Academic Press, San Diego, Calif, USA, 1999. 
[7] E. F. Doungmo Goufo and S. C. Oukouomi Noutchie, "Honesty in discrete, nonlocal and randomly position structured fragmentation model with unbounded rates," Comptes Rendus Mathematique, vol. 351, no. 19-20, pp. 753-759, 2013.

[8] E. F. Doungmo Goufo and S. C. Oukouomi Noutchie, "Global analysis of a discrete nonlocal and nonautonomous fragmentation dynamics occurring in a moving process," Abstract and Applied Analysis, vol. 2013, Article ID 484391, 9 pages, 2013.

[9] S. C. Oukouomi Noutchie and E. F. Doungmo Goufo, "Global solvability of a continuous model for nonlocal fragmentation dynamics in a moving medium," Mathematical Problems in Engineering, vol. 2013, Article ID 320750, 8 pages, 2013.

[10] S. C. Oukouomi Noutchie and E. F. Doungmo Goufo, "On the honesty in nonlocal and discrete fragmentation dynamics in size and random position," ISRN Mathematical Analysis, vol. 2013, Article ID 908753, 7 pages, 2013.

[11] W. Wagner, "Explosion phenomena in stochastic coagulationfragmentation models," The Annals of Applied Probability, vol. 15, no. 3, pp. 2081-2112, 2005.

[12] R. Blatz and J. N. Tobobsky, "Statistical investigation of fragments of polymer molecules," The Journal of Physical Chemistry, vol. 49, p. 77, 1945.

[13] H. Mark and R. Simha, "Degradation of long chain molecules," Transactions of the Faraday Society, vol. 35, pp. 611-618, 1940.

[14] R. M. Ziff and E. D. McGrady, "The kinetics of cluster fragmentation and depolymerisation," Journal of Physics A, vol. 18, no. 15, pp. 3027-3037, 1985.

[15] M. Caputo, "Linear models of dissipation whose $\mathrm{Q}$ is almost frequency independent," Journal of the Royal Australian Historical Society, vol. 13, part 2, pp. 529-539, 1967.

[16] I. Podlubny, "Fractional-order systems and $P I^{\lambda} \mathrm{D}^{\mu}$ controllers," IEEE Transactions on Automatic Control, vol. 44, no. 1, pp. 208214, 1999.

[17] J. Spanier and K. B. Oldham, An Atlas of Functions, Hemisphere (Subsidiary of Harper \& Row), New York, NY, USA, 1987.

[18] T. T. Hartley and C. F. Lorenzo, "A solution to the fundamental linear fractional order differential equation," Tech. Rep. NASA/TP-1998-208963, 1998. 


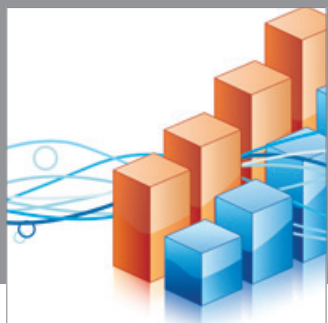

Advances in

Operations Research

mansans

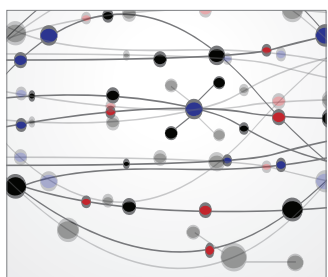

The Scientific World Journal
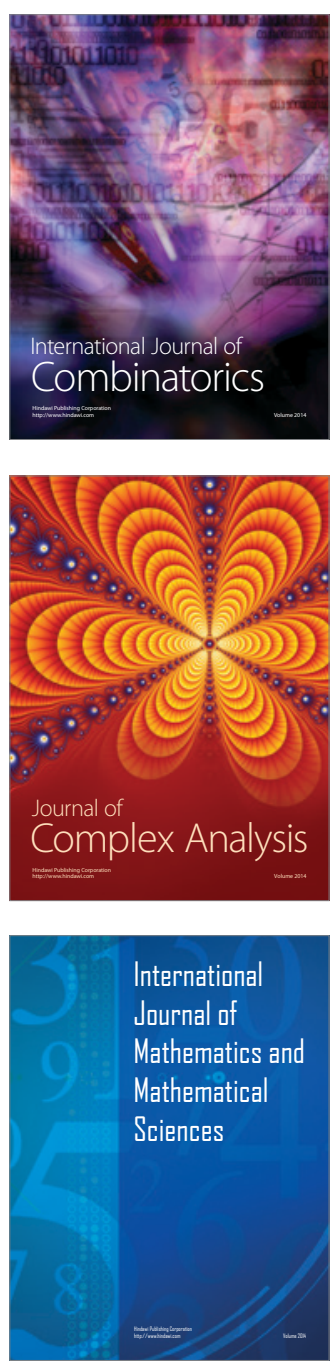
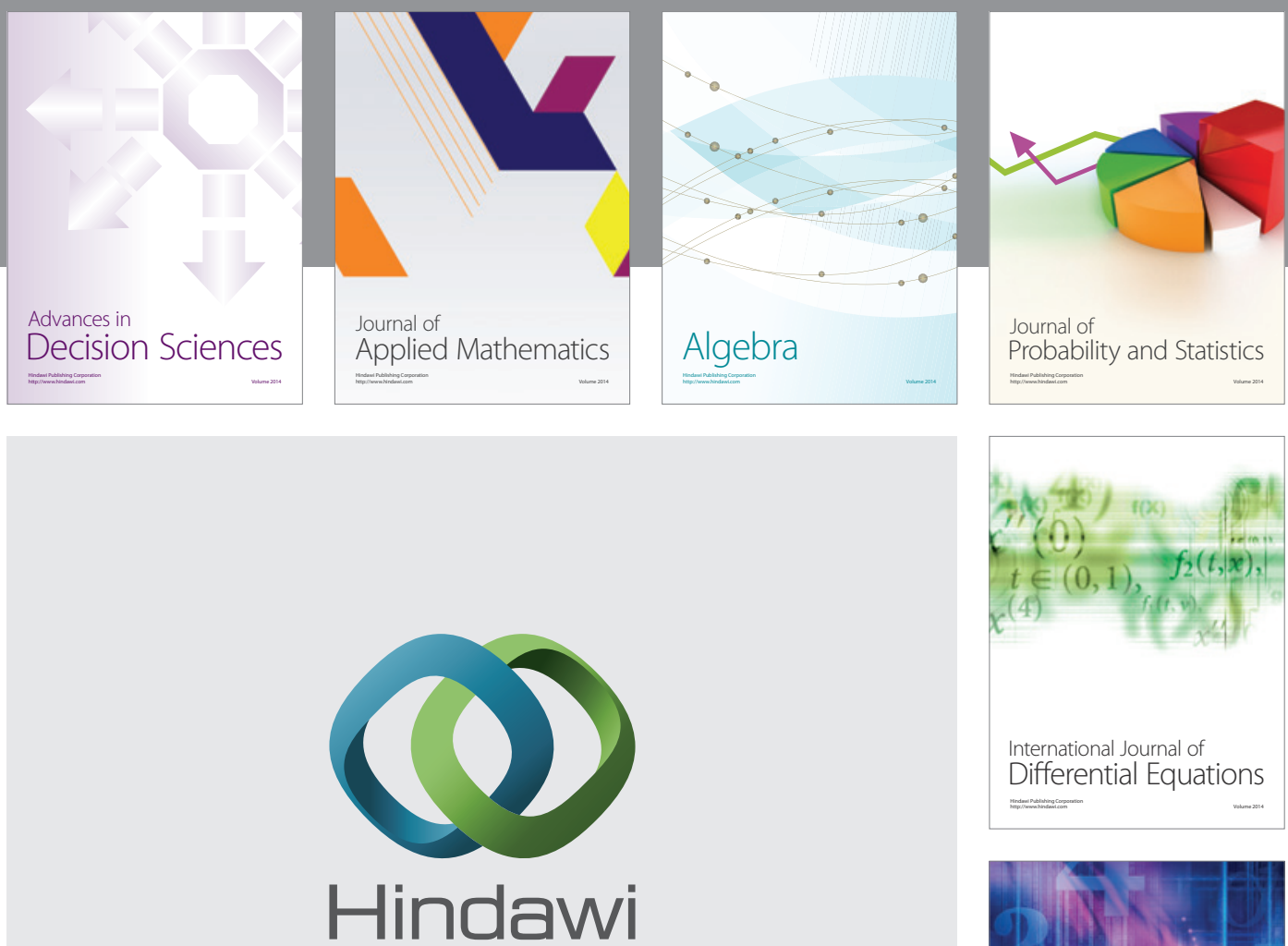

Submit your manuscripts at http://www.hindawi.com
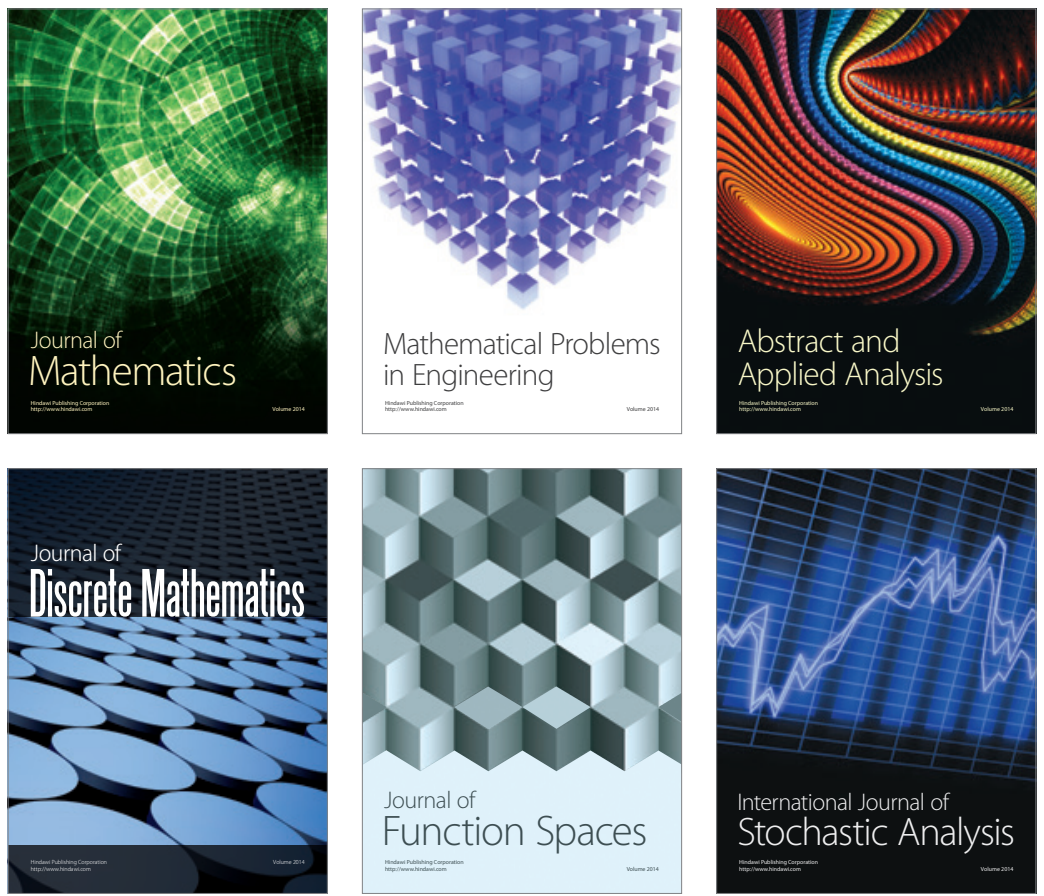

Journal of

Function Spaces

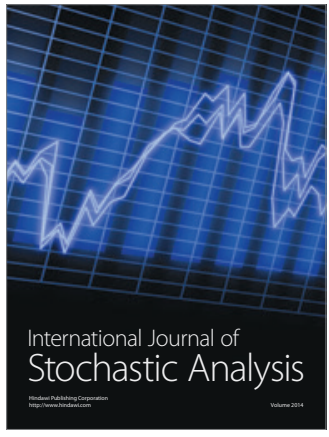

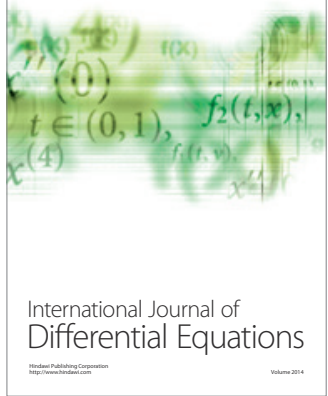
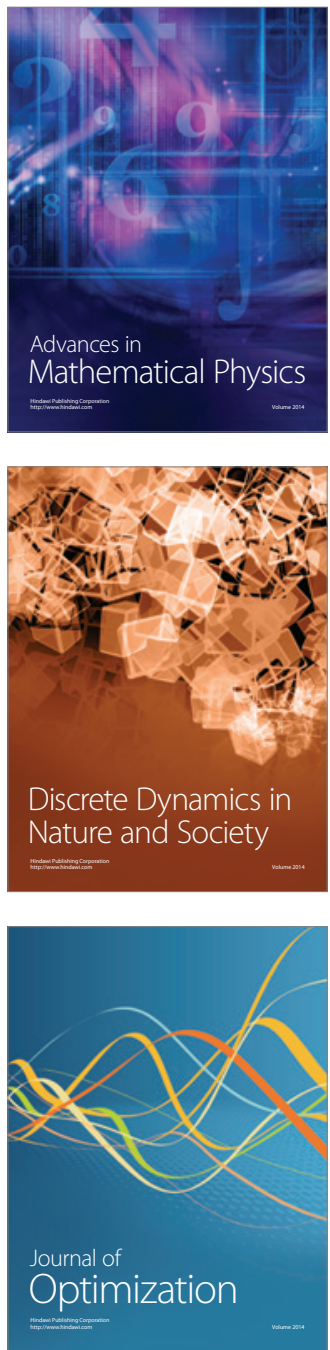